\title{
AN INVESTIGATION OF MEASURING DRIVER ANGER WITH ELECTROMYOGRAPHY
}

\author{
Christopher Saikalis, John Cliburn, Cedric Portea, Yi-Ching Lee \\ George Mason University \\ Fairfax, Virginia, USA \\ Email: ylee65@gmu.edu
}

\begin{abstract}
Summary: This research explores a novel approach to measuring driver anger using facial electromyography (EMG) while completing a navigation task on a driving simulator. Participants' anger was induced by traffic events that were frustrating in nature as well as time pressure while having to follow navigational directions. Participants' feeling of anger was assessed multiple times via subjective self-reports while being continuously monitored through a facial EMG. Participants' trait driving anger was assessed using the Driving Anger Scale. Results showed that, compared to baseline measures, participants had significantly higher facial EMG activation values and subjective feelings of anger upon experiencing frustrating events, suggesting facial EMG as a reliable physiological measurement for inferring drivers' feelings of anger. This experimental protocol can be used to assess anger in navigational contexts in future studies.
\end{abstract}

\section{INTRODUCTION}

Anger has a vast body of supporting evidence for its effects on driving such as driving at higher speeds, maintaining shorter distance and time headway behind a lead vehicle, and showing greater variations in lateral positions (Zhang, Chan, Ba, \& Zhang, 2016). Angry drivers can be more careless and less well-prepared and have a narrower scanning area (Zhang et al., 2016). Research has shown that anger while driving appears to be reactive to the environment (Deffenbacher, Huff, Lynch, Oetting, \& Salvatore, 2000) where a series of frustrating events in the driving environment may prompt drivers to drive more aggressively even if they are not aggressive ordinarily (Abou-Zeid, Kaysi, \& Al-Naghi, 2011). Also, internal characteristics such as a driver's trait anger influences the degree to which they react aggressively to frustrating events in the traffic environment (Abou-Zeid et al., 2011).

Understanding the effects of a driver's affective state is more important than ever as about 9 in 10 drivers perceived aggressive driving to be a serious threat to their safety and $78 \%$ of drivers have reported engaging in at least one aggressive driving behavior over the last year (AAA Foundation for Traffic Safety, 2016). The current research aimed to measure driver anger using physiological measures, the results of which may be beneficial as we look to mitigate the consequences of a negative affective state. Facial electromyography (EMG) is a method that has been used to track emotions over time; this technique provides many advantages over self-report measures (Hazlett \& Benedek, 2007), such as the ability to measure emotional valence over time without having to rely on self-reports of emotions. One of the primary facial muscles associated with negative mood, frustration, and anger, is the corrugator supercilia, which is also responsible for producing frowns (Heller et al., 2011; Van Boxtel, 2010). The goal of this research was to investigate the relationship between facial EMG and driver anger. The results of this research 
may help lay a foundation for future studies to refine methods and standardize this approach to measure driver affective state.

This initial proof-of-concept study investigated three hypotheses. Hypothesis 1 was that upon experiencing frustrating events, participants would report higher levels of subjective anger when compared to baseline. Hypothesis 2 anticipated that participants who scored higher on trait driving anger would experience greater feelings of anger upon experiencing frustrating events than participants with lower trait driving anger. Hypothesis 3 was that corrugator supercilia activation would increase during the frustrating events drive when compared to baseline.

\section{METHODS}

\section{Participants}

Eleven participants, 7 male and 4 female, were recruited and participated in this study. Participants were recruited through the Opportunities for Research Participants (Sona Systems) in the Department of Psychology at George Mason University. The mean participant age was 21 $(\mathrm{SD}=7.1)$ years. The mean years of driving experience was 5.4 years $(\mathrm{SD}=7.3)$. Participants were compensated with class credit for their participation.

\section{Apparatus}

The driving simulator apparatus was a Realtime Technologies desktop simulator comprised of driver's seat, pedal apparatus, and a Logitech steering wheel. The simulation was displayed on a 1920x1080 desktop monitor. The simulated environment was built with SimVista and SimCreator software. The driving environment was composed of rural and urban settings with variations of buildings, trees, curves, intersections, and construction zones. In total, participants completed three different drives: the training drive allowed the participant time to familiarize themselves with the driving simulator and the simulated driving environment and two experimental drives - a control drive and an event drive (see details of the experimental drives below). The order of the experimental drives was counter-balanced to prevent order effects. Facial electromyography (EMG) data were collected via a BIOPAC MP 150 system with the Electromyogram Amplifier (EMG100C). The EMG was set to a sample rate of 1000/second, gain at 2000, and 100HzHP (OFF) \& 500HzLP. The electrodes used were BIOPAC EL513, they are disposable cloth electrodes designed for facial EMG. EMG data were collected and processed using AcqKnowledge 4.3 software. Two shielded electrodes were placed on the area of the corrugator supercilia (+/-) and a ground electrode placed just below the cheekbone, in accordance with Fridlund's research procedure (1986).

The short form of Driving Anger Scale (DAS) (Deffenbacher, Oetting, \& Lynch, 1994) was used to measure the general trait driving anger (14-item on a scale of 1-5, reliability of .80). DAS scores represent the likelihood of risky driving behavior, with higher scores leading to an increased chance of risky driving behavior (Deffenbacher et al., 1994). The Discrete Emotions Questionnaire (DEQ) (Harmon-Jones, Bastian, \& Harmon-Jones, 2016) was a short measure of basic emotions such as anger, disgust, fear, sadness, and happiness on a scale of 1-7. The DEQ was given to participants four times. This assessment throughout the study served as a 
manipulation check. A demographic questionnaire was used to collect participant age, gender, and years of driving experience. A motion sickness screening questionnaire was used to screen out participants who may be susceptible to experiencing motion sickness symptoms.

\section{Anger Induction}

We aimed to induce anger by using a frustrating driving scenario where the driver must follow navigational directions while under time pressure and encountering frustrating events. This approach is similar to that of Lee and colleagues $(2010,2016)$. This induction approach previously showed that drivers who had more negative emotions had more unsafe reactions to frustrating events (Lee, 2010), and we theorized that with repeated frustrating events, the feelings of negative emotions (e.g., anger) would be compounded. Twelve frustrating events were programed into the event drive to induce feelings of anger. Some events participants encountered included: a randomly braking lead vehicle, a person unexpectedly crossing the road, cars suddenly pulling out in front of the driver, and instances of heavy traffic. These events were previously tested and validated by Lee and Winston (2016). The frustrating events were placed throughout the entire event drive but occurred more frequently towards the beginning of the drive to invoke an effect earlier in the experiment. For comparison purposes, the control drive had identical road network, ambient traffic, and infrastructure as the event-drive. This control drive did not have any frustrating events. Both drives took about 10 minutes to complete, if driven at $20 \mathrm{mph}$.

Navigational directions were provided to participants in the form of a directional arrow in the bottom right of the simulator display, the black arrow would appear in the white box shown in the right image of Figure 1. The placement in the bottom right corner of the display was chosen as representative of where a driver's GPS system may likely be located in reference to forward traffic scene in a real-life driving situation. Turn by turn directions were provided when participants approached intersections. The directions provided the next turn to be made by the participant, leading them on a preprogrammed path. Construction cones and detour signs were also used to further delineate the preprogrammed path. The control and event drives each contained one correct route from start to finish. Alternate routes were built into the environment which would allow the participant to recover from a missed turn.

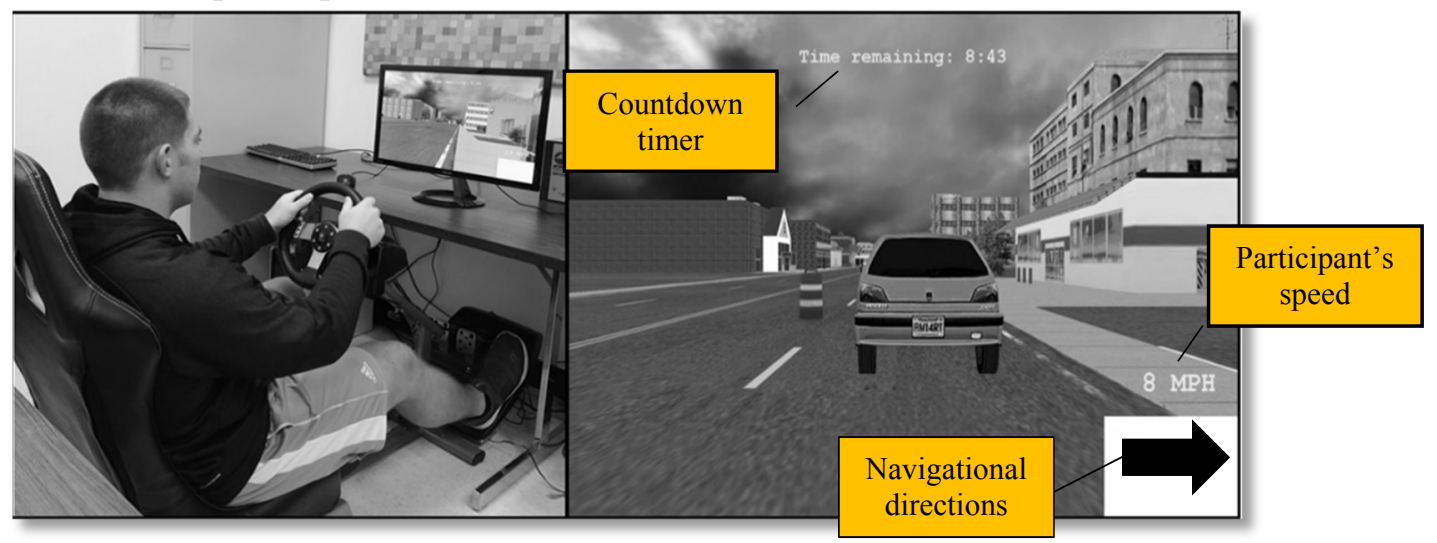

Figure 1. Configurations of driving simulator and scenarios - left image is the driving simulator apparatus and right image is an example of a slow-moving lead vehicle frustrating event. The countdown clock, driver's current speed in miles per hour, and the navigational directions are depicted 
Time pressure was manipulated via a countdown timer shown at the top of the simulator display (see Figure 1). The timer represented the amount of time remaining for the participant to reach the destination. The goal of this timer was to create a sense of urgency for completing the experimental drives. The timer started when each drive began and showed the initial amount of time of 10 minutes. If participants did not finish the drives within 10 minutes, a message would appear at the top of the screen notifying the participant to stop driving.

\section{Procedure}

Upon entering the lab, participants were directed to a seat at a desk where they were asked to read and sign a consent form. Then, participants were verbally administered a motion sickness screening questionnaire, and completed DAS and the demographic questionnaire. Upon completion of the self-reports, participants were asked to move to the driver's seat of the simulator. The electrodes for the EMG were then attached to their respective locations on the participants' faces. A 2-minute baseline EMG recording was conducted which served as the participants baseline. The participants then completed the DEQ. Participants were read the instructions and proceeded to complete the training drive, which lasted for 5 minutes. Included in the instructions, participants were told to assume they are driving in an unfamiliar place, to abide by all traffic laws, avoid collisions, and the speed limit was $20 \mathrm{mph}$. Following the training drive, participants completed the DEQ and then began the first experimental drive. For participants in condition 1, the control drive was completed first followed by the event drive. For participants in condition 2, the event drive was completed first followed by the control drive. In total, the DEQ was completed four times: before and after the training drive, after first experimental drive, and finally after second experimental drive. The entirety of the driving sections of the experiment was performed on a desktop driving simulator. On average, participants took 40-50 minutes to complete the experiment.

\section{Analytic plans}

The dependent variables of this research were trait driving anger, facial EMG values, and selfreport feelings of anger. R studio, version 1.1.383, was used to run all statistical analyses. EMG values were analyzed using AcqKnowledge 4.3, the raw EMG was processed using the root mean square at an interval of 0.03 seconds.

\section{RESULTS}

\section{EMG Activation}

The analysis for the EMG data was done in two steps. First, a paired-samples t-test was conducted to compare the average baseline activation to the average activation from the event drive. The average activation from the event drive $(\mathrm{M}=0.0062, \mathrm{SD}=0.00098)$ was significantly higher than the average baseline $(\mathrm{M}=0.0055, \mathrm{SD}=0.00042)$ activation, $t(10)=-2.83, p=0.01$. Second, individual facial EMG activity was standardized and expressed as the percentage change between event drive and baseline. The average EMG value for each participant during the baseline and event drive can be seen in Figure 2. On average, corrugator supercilia activity 
increased by $10.4 \%$ during the event drive $(\mathrm{SD}=11.5 \%)$, suggesting the association between increased corrugator supercilia activity and frustrating events during the event drive.

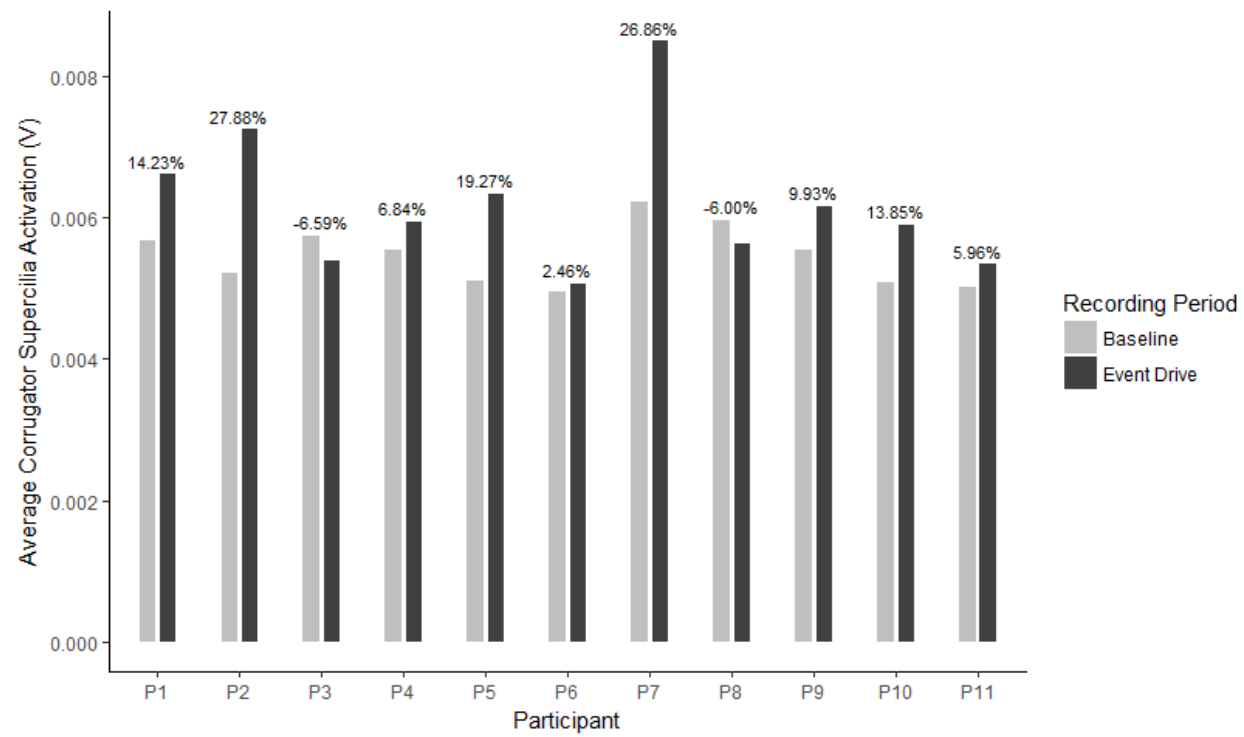

Figure 2. Percentage change of corrugator supercilia activity from baseline and event drive

\section{Self-reported Driver Anger}

A one-way repeated measures ANOVA was conducted to compare the reported emotions on DEQ throughout the experiment. The results showed that there was a significant difference in at least two time points, $F(3,30)=4.43, p<.05$. Mauchly-s test indicated a violation of sphericity, so degrees of freedom were corrected using Greenhouse-Geisser estimates of sphericity.

Correcting for sphericity still produced a significant difference between at least 2 time points, $p<$ .05. Post hoc paired t-test revealed that anger DEQ scores were not significantly different from pre-training $(\mathrm{M}=4.2, \mathrm{SD}=0.6)$, to post-training $(\mathrm{M}=4.8, \mathrm{SD}=2.4)$, and to post-control $(\mathrm{M}=$ $4.7, \mathrm{SD}=1.3)$. However, there was a significant difference on anger DEQ scores between postcontrol drive $(\mathrm{M}=4.7, \mathrm{SD}=1.3)$ and post-event drive $(\mathrm{M}=6.2, \mathrm{SD}=2.5), t(10)=-2.95, p=.01$ (Figure 3), meaning that the reported anger increased from 4.7 to 6.2 on a 7 -point scale between these two time points.

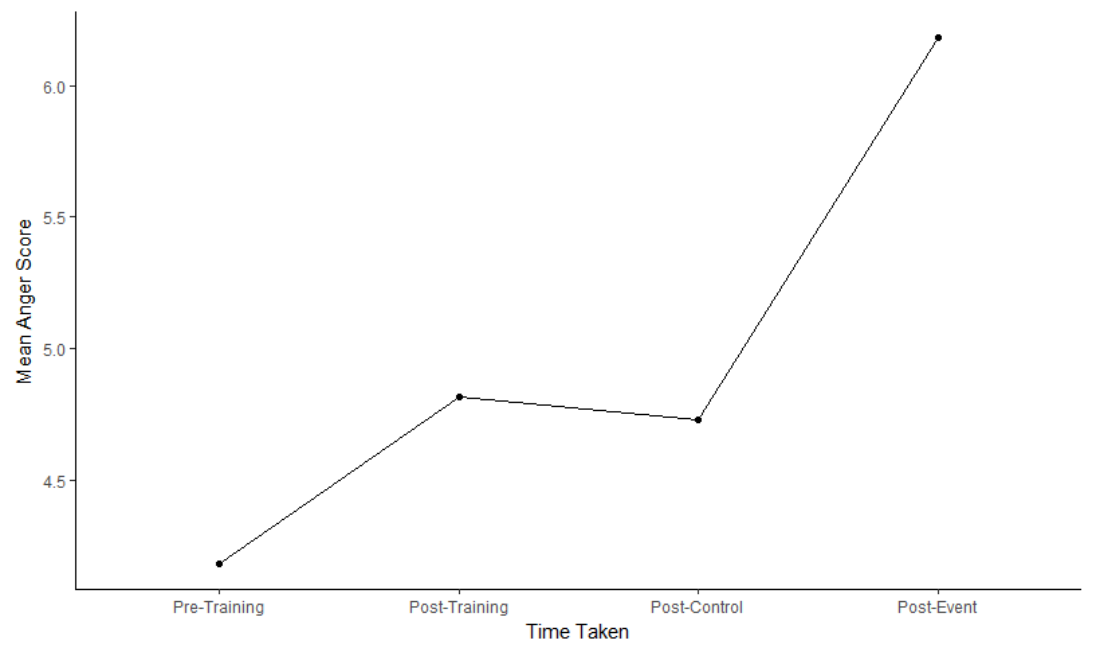

Figure 3. Mean anger scores for four time points 


\section{Driving Performance and DAS}

Participants' reported anger (DEQ) following the event drive was used to predict headway distance to a lead vehicle using a linear regression. A significant regression equation was found, $F(1,9)=5.24, p<.05$, with an adjusted $\mathrm{R}^{2}$ of 0.30 , indicating that higher anger score predicted shorter headway distance. DAS scores did not predict the reported anger (DEQ) after the event drive, $p>.05$, suggesting that participants' trait anger did not predict their self-reported feelings of anger after experiencing the event drive.

\section{CONCLUSION}

The current study aimed to measure driver anger using facial EMG during a navigational driving task. The three hypotheses under investigation predicted that 1) participants would report higher levels of subjective anger when compared to baseline after experiencing frustrating events, 2) participants who scored higher on trait driving anger would experience greater feelings of anger upon experiencing frustrating events than participants with lower trait driving anger, and 3) corrugator supercilia activation would increase during the event drive when compared to baseline. Both subjective and objective measures of anger indicated that the anger manipulations were successful in putting participants in a negative affective state, thus confirming hypothesis 1. Hypothesis 2 was not supported: there was not a significant relationship between trait driving anger and self-reported feeling of anger upon experiencing the frustrating events.

The results from our EMG analysis confirmed our $3^{\text {rd }}$ hypothesis; there was a significant increase in corrugator supercilia activity during the event drive when compared to baseline.

The change in activations between the baseline drives and event drives indicated that the combinations of frustrating events, time pressure, and navigational directions led to significant effects on driver's state. We suspect that the increase in activation during the entire event drive was due to a combination of the events compounding over time and the added time pressure.

Even though the sample size was small, the results indicated potentials in using facial EMGs to infer the affective state (anger) of drivers. The small sample size could be one reason why hypothesis 2 was not supported. Findings from this initial proof-of-concept study suggest that induction methods that combine dynamic traffic events and time pressure manipulations can be used to examine driver's affective states in a simulator-based navigational context. This experimental protocol should be further validated with a larger sample size but has the potential to be used in other contexts where drivers' internal and external stressors may influence their emotional state.

\section{ACKNOWLEDGMENTS}

The authors wish to acknowledge the assistance and support from members of the Health Behaviors Lab at George Mason University. This work was supported by the National Science Foundation (Award \# 1653624). 


\section{REFERENCES}

AAA Foundation for Traffic Safety. (2016, July). Prevalence of Self-Reported Aggressive Driving Behavior: United States, 2014. Retrieved July 11, 2018, from https://newsroom.aaa.com/wpcontent/uploads/2016/07/aggressive_driving_REPORT_7_14.pdf

Abou-Zeid, M., Kaysi, I., \& Al-Naghi, H. (2011). MEASURING AGGRESSIVE DRIVING BEHAVIOR USING A DRIVING SIMULATOR: AN EXPLORATORY STUDY.

Deffenbacher, J. L., Huff, M. E., Lynch, R. S., Oetting, E. R., \& Salvatore, N. F. (2000). Characteristics and treatment of high-anger drivers. Journal of Counseling Psychology, 47(1), 5-17. https://doi.org/10.1037/0022-0167.47.1.5

Deffenbacher, J. L., Oetting, E. R., \& Lynch, R. S. (1994). Development of a Driving Anger Scale. Psychological Reports, 74(1), 83-91. https://doi.org/10.2466/pr0.1994.74.1.83

Fridlund, A. J., \& Cacioppo, J. T. (n.d.). Guidelines for Human Electromyographic Research. Psychophysiology, 23(5), 567-589. https://doi.org/10.1111/j.1469-8986.1986.tb00676.x

Harmon-Jones, C., Bastian, B., \& Harmon-Jones, E. (2016). The Discrete Emotions Questionnaire: A New Tool for Measuring State Self-Reported Emotions. PLOS ONE, 11(8), e0159915. https://doi.org/10.1371/journal.pone.0159915

Hazlett, R. L., \& Benedek, J. (2007). Measuring emotional valence to understand the user's experience of software. International Journal of Human-Computer Studies, 65(4), 306-314. https://doi.org/10.1016/j.ijhcs.2006.11.005

Heller, A. S., Greischar, L. L., Honor, A., Anderle, M. J., \& Davidson, R. J. (2011). Simultaneous acquisition of corrugator electromyography and functional magnetic resonance imaging: A new method for objectively measuring affect and neural activity concurrently. NeuroImage, 58(3), 930-934. https://doi.org/10.1016/j.neuroimage.2011.06.057

Lee, Y.-C. (2010). Measuring Drivers' Frustration in a Driving Simulator. Proceedings of the Human Factors and Ergonomics Society Annual Meeting, 54(19), 1531-1535. https://doi.org/10.1177/154193121005401937

Lee, Y.-C., \& Winston, F. K. (2016). Stress induction techniques in a driving simulator and reactions from newly licensed drivers. Transportation Research Part F: Traffic Psychology and Behaviour, 42(Part 1), 44-55. https://doi.org/10.1016/j.trf.2016.06.019

van Boxtel, A. (2010). Facial EMG as a Tool for Inferring Affective States, 5.

Zhang, T., Chan, A. H. S., Ba, Y., \& Zhang, W. (2016). Situational driving anger, driving performance and allocation of visual attention. Transportation Research Part F: Traffic Psychology and Behaviour, 42(Part 2), 376-388. https://doi.org/10.1016/j.trf.2015.05.008 